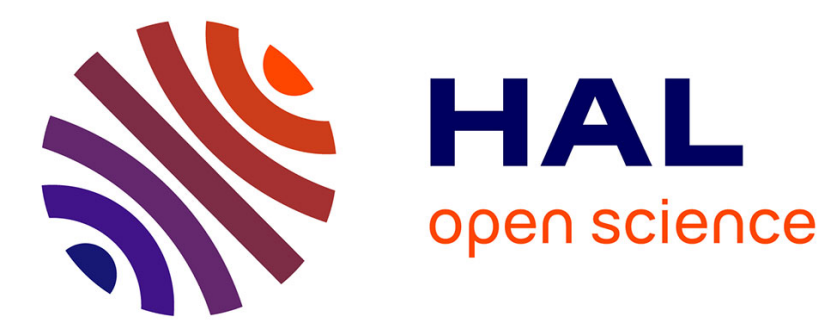

\title{
Experimental test of unsteady Stokes' drag force on a sphere
}

\author{
Jacques Vigué, Gilles Dolfo, D. Lhuillier
}

\section{To cite this version:}

Jacques Vigué, Gilles Dolfo, D. Lhuillier. Experimental test of unsteady Stokes' drag force on a sphere. Experiments in Fluids, 2020, 61 (4), pp.97. 10.1007/s00348-020-2936-6 . hal-02499772

\section{HAL Id: hal-02499772}

\section{https://hal.science/hal-02499772}

Submitted on 5 Mar 2020

HAL is a multi-disciplinary open access archive for the deposit and dissemination of scientific research documents, whether they are published or not. The documents may come from teaching and research institutions in France or abroad, or from public or private research centers.
L'archive ouverte pluridisciplinaire HAL, est destinée au dépôt et à la diffusion de documents scientifiques de niveau recherche, publiés ou non, émanant des établissements d'enseignement et de recherche français ou étrangers, des laboratoires publics ou privés. 


\title{
Experimental test of unsteady Stokes' drag force on a sphere
}

\author{
G. Dolfo · J. Vigué · D. Lhuillier
}

Received: date / Accepted: date

\begin{abstract}
Stokes calculated the force exerted by the surrounding fluid on a sphere and on a cylinder in oscillating motion. Although these results are valid only if the Reynolds number $R e$ is very small, $R e \ll 1$, all the tests on macroscopic spheres have been made with $R e$ larger than 20. Here, we describe an experiment which measures the drag force on an oscillating sphere with small values of the Reynolds number, down to $R e \approx 0.03$ for the smallest sphere studied here while the Stokes' number $S t$ is large, between 150 and 1500. Our measurements are in very good agreement with Stokes' result and, in particular, they exhibit the quadratic dependence of the force with the sphere radius when this radius is larger than the viscous penetration depth $\delta$.
\end{abstract}

Keywords First keyword · Second keyword · More

\section{Introduction}

The damping of a pendulum oscillating in air by the drag force is a very classic experiment and, in the case of a sphere or a cylinder, this force has been calculated in 1851 by Stokes [1]. In the case of an oscillatory

\section{G. Dolfo}

Laboratoire Collisions Agrégats Réactivité-IRSAMC, Université de Toulouse-UPS and CNRS UMR 5589, Toulouse,

France E-mail: gilles.dolfo@wanadoo.fr

\section{J. Vigué}

Laboratoire Collisions Agrégats Réactivité-IRSAMC, Université de Toulouse-UPS and CNRS UMR 5589, Toulouse, France E-mail: jacques.vigue@irsamc.ups-tlse.fr

D. Lhuillier

Sorbonne Université, CNRS, Institut Jean Le Rond d'Alembert, Paris, France

E-mail: daniel.lhuillier@upmc.fr motion, the drag force on a sphere becomes a quadratic function of the sphere radius $a$ if $a$ is considerably larger than the viscous penetration depth $\delta$ defined below and, in this case, the drag force is considerably larger than the drag force on the same sphere with the same velocity in steady motion. This classic result can be found, for example, in the Landau and Lifschitz book "Fluid mechanics" [2]. However, all the tests of this drag force on spheres have been made with large values of the Reynolds number $R e \geq 20$ while the validity of Stokes calculation requires $R e \ll 1$.

In this paper, we present the measurement of the air drag force on a series of pendulums made of a sphere suspended by a thin wire. To reach the Stokes' regime, it is necessary to measure very small oscillations and we use a shadow detector, with a sub-micrometer sensitivity. We thus measure the damping time constant of the oscillations with the pendulum oscillating either in air at atmospheric pressure or under a very low pressure, this latter experiment measuring the nonhydrodynamic friction forces. We then deduce the drag force from the calculated pendulum energy. Our results are in very good agreement with Stokes' result and, in particular, with the quadratic dependence on the sphere radius $a$. Our analysis takes into account the drag force on the wire and the modification of the drag force on the sphere due the confinement of the air by the vessel enclosing the pendulum.

The content of the paper is organized as follows: we recall Stokes' results in section 2 and previous tests of this force in section 3. Section 4 describes our experiment and section 5 the data analysis. A simplified calculation of the connection between the drag force and the damping time constant is presented in section 6 . The modification of this force due to fluid confinement is discussed in section 7 . The measurements of 
this force are presented and discussed in section 8. A brief conclusion, section 9, summarizes our results and three appendices give complementary information.

\section{Stokes' results}

2.1 Some general ideas and Stokes' results

When an object moves in an incompressible fluid, the fluid motion is described by Navier-Stokes equations [2] which relate the velocity $\mathbf{v}$ and the pressure $p$

$$
\begin{aligned}
\rho\left(\frac{\partial \mathbf{v}}{\partial t}+(\mathbf{v} \cdot \nabla) \mathbf{v}\right) & =-\nabla p+\eta \nabla^{2} \mathbf{v}, \\
\nabla \cdot \mathbf{v} & =0
\end{aligned}
$$

where $\rho$ is the fluid density and $\eta$ its viscosity. The force exerted by the fluid on this object generally depends on two dimensionless numbers, the Reynolds number $R e$ and the Stokes' number $S t$, symbolically defined as

$R e \sim \frac{|\rho(\mathbf{v} \cdot \nabla) \mathbf{v}|}{\left|\eta \nabla^{2} \mathbf{v}\right|}, \quad S t \sim \frac{|\rho(\partial \mathbf{v} / \partial t)|}{\left|\eta \nabla^{2} \mathbf{v}\right|}$.

The immersed object we have in mind is a pendulum made of a wire fixed at one end while its moving end is attached to a sphere (see Fig. 1). Let us consider the forces exerted on the two parts of the pendulum.

We consider a sphere of radius $a$, oscillating along the $\mathbf{x}$-axis, with its center at $x(t)=x_{0} \cos (\omega t)$ and then

$R e \sim \frac{\rho a x_{0} \omega}{\eta}, \quad S t \sim \frac{\rho a^{2} \omega}{\eta}$.

The force exerted on the sphere was calculated, in the limit of zero Reynolds number, by Stokes. This force, parallel to the $\mathbf{x}$-axis, is the sum of a drag force and an added-mass force $[1,2]$

$F_{x}=-6 \pi \eta a\left(1+\frac{a}{\delta}\right) \frac{d x}{d t}-\frac{2 \pi}{3} \rho a^{3}\left(1+\frac{9 \delta}{2 a}\right) \frac{d^{2} x}{d t^{2}}$,

where $\delta=\sqrt{2 \eta / \rho \omega}$ is the viscous penetration depth. As could be anticipated, this $R e=0$ force only depends on the Stokes' number $S t \sim a^{2} / \delta^{2}$.

For the pendulum wire, we use Stokes' results for an infinitely long cylinder of radius $R$ with its axis parallel to the $\mathbf{z}$-axis oscillating along the $\mathbf{x}$-axis with its center at $x(t)=x_{0} \cos (\omega t)$. In this case, the Reynolds number $R e$ and the Stokes' number $S t$ are given by

$R e \sim \frac{\rho R x_{0} \omega}{\eta}, \quad S t \sim \frac{\rho R^{2} \omega}{\eta} \propto \frac{R^{2}}{\delta^{2}}$.

Stokes also calculated the $R e=0$ force per unit length of the cylinder $d F_{x} / d l$

$\frac{d F_{x}}{d l}=-2 \pi \eta\left[\left(\frac{R}{\delta}\right)^{2} k^{\prime} \frac{d x}{d t}+\frac{1}{\omega}\left(\frac{R}{\delta}\right)^{2} k \frac{d^{2} x}{d t^{2}}\right]$.
The quantities $k$ and $k^{\prime}$ were given in closed form by Stuart in 1963 [3] (see also [4])

$k-i k^{\prime}=1+\frac{4 K_{1}(Z)}{Z K_{0}(Z)}$,

where $K_{0}$ and $K_{1}$ are modified Bessel functions and $Z=(1+i) R / \delta$. The quantities $R^{2} k / \delta^{2}$ and $R^{2} k^{\prime} / \delta^{2}$ vary slowly with the ratio $R / \delta$ and tend toward 0 when $R / \delta \rightarrow 0$ while $k$ and $k^{\prime}$ diverge.

\subsection{Validity of these results}

Equation (3) defines the Reynolds and Stokes numbers up to some undetermined proportionality constant. Since we want to compare our results to those of previous works, we adopt for the sphere the widely used definitions

$R e=\frac{4 x_{0} a}{\delta^{2}} \quad, \quad S t=\frac{4 a^{2}}{\delta^{2}}$

Stokes' result, eq. (4), was obtained for $R e=0$ and arbitrary values of the Stokes' number $S t$. In our experiments, we will strive to satisfy the condition $x_{0} \ll$ $a$ (which severely limits the amplitude of oscillations) so as to have very small Reynolds number while the Stokes' number will be large.

Stokes' calculation involves a second approximation: the boundary conditions were written at the mean position of the oscillating sphere, which is a good approximation if the amplitude verifies $x_{0} / a \ll 1$ and $x_{0} / \delta \ll 1$. These dimensionless ratios [5] can be written as a function of $R e$ and $S t$

$\frac{x_{0}}{a}=\frac{R e}{S t}$ and $\frac{x_{0}}{\delta}=\frac{R e}{2 \sqrt{S t}}$.

If the conditions $x_{0} / a \ll 1$ and $x_{0} / \delta \ll 1$ are not fulfilled, the force is no more proportional to the oscillation amplitude, as shown by Berg et al. [7] with measurements and numerical calculations for an oscillating cylinder: the additional force increases with the ratio $x_{0} / \delta$. We are not aware of a similar work for spheres.

For the wire treated as a cylinder of radius $R$, we define the Reynolds and Stokes numbers in the same way

$R e_{C}=\frac{4 x_{0} R}{\delta^{2}}, \quad S t_{C}=\frac{4 R^{2}}{\delta^{2}}$

and the discussion is similar. Because the cylinder radius $R$ is considerably smaller that the sphere radius $a$ $R e_{C} \ll R e$ and $S t_{C} \ll S t$.

\subsection{Our choice}

We chose to measure the drag force on an oscillating sphere: this force is equal to the force in a steady 
motion, $F_{x}=-6 \pi \eta a d x / d t$, multiplied by the factor $[1+(a / \delta)]$. This multiplying factor, equal to $[1+\sqrt{S t} / 2]$, is quite large, up to $\approx 21 \mathrm{in}$ our experiments. We thought it interesting to test this somewhat surprising behavior.

\section{Experimental tests of the force on an oscillating object}

\subsection{Tests with macroscopic objects}

These tests have been done with different types of systems:

- a gravity pendulum with a cylindrical or spherical body [8-11];

- a string vibrating in air or in a liquid $[12,13]$;

- a torsion pendulum oscillating in a liquid [14-16] or in air [17];

- a microsphere oscillating at various temperatures in liquid helium, using magnetic levitation in a superconducting capacitor [18].

These different experiments investigate different ranges of the radius $a$ and of the viscous penetration depth $\delta \propto \sqrt{1 / \omega}$. Many other papers have studied the damping of a pendulum but their experimental results are not compared to Stokes' results. We may have missed old works not quoted by recent papers. Table 1 summarizes the measurements of the drag force on spheres. The results of Jäger et al. [18], reanalyzed by Bolster et al. [11], have not been included because some information is missing: following Bolster et al., the Reynolds number is larger than 20 (respectively 40) when the helium temperature is $2.1 \mathrm{~K}$ (respectively $2.2 \mathrm{~K}$ ) in these experiments. As far as we know, no experiment with oscillating spheres has explored Reynolds numbers smaller than about 20 .

With a macroscopic sphere or cylinder oscillating in air, Stokes' regime, $R e \ll 1$, is difficult to observe : for a radius equal to $5 \mathrm{~mm}, R e=1$ corresponds to a velocity equal to $1.5 \mathrm{~mm} / \mathrm{s}$ and, if the frequency is close to $1 \mathrm{~Hz}$, to an amplitude $x_{0} \approx 0.25 \mathrm{~mm}$. The condition $R e \ll 1$ is verified only by very small values of $x_{0}$ and it is necessary to measure very small displacements.

\subsection{Tests with microscopic particles}

Microrheology methods, which have developed during the last forty years, are reviewed by Gardel et al. [19]. These experiments use micron-sized probe particles and very sensitive position-measurement techniques. Some experiments are active, with the application of forces or torques on the probe particle, while other experiments
Table 1 The information collected in this table is taken from the papers of Baily [8], Gupta et al. [10], Bolster et al. [11]. From the published data, whenever it was necessary, we have calculated the values of the angular frequency $\omega$ and of the viscous penetration depth $\delta$, the Stokes number $S t$, the minimum and maximum values of the oscillation amplitude $x_{0}$ and of the Reynolds number Re during the experiments (all the lengths are measured in $\mathrm{mm}$ ). Baily describes numerous experiments and the values given in this table corresponds to average values of the oscillation amplitudes of the experiments performed at atmospheric pressure. The paper of Bolster et al. describes the experiments with two pendulum lengths and we reproduce here only the data obtained with the longest pendulum. The minimum amplitude $x_{0 \text { min }} \sim 1 \mathrm{~mm}$ is taken from figure $4 \mathrm{~A}$ of this paper. In the papers of Gupta et al. and Bolster et al., the authors give the maximum Reynolds number for which the damping force is a linear function of the velocity and we have used this value as $R e_{\max }$.

\begin{tabular}{|c|c|c|c|c|c|c|c|}
\hline Author & \multicolumn{2}{|c|}{ Baily } & \multicolumn{2}{c|}{ Gupta et al. } & \multicolumn{3}{c|}{ Bolster et al. } \\
\hline Fluid & \multicolumn{2}{|c|}{ air } & \multicolumn{2}{c|}{ air } & \multicolumn{3}{c|}{ water } \\
\hline$a$ & 37 & 52 & 165 & 224 & 12.7 & 19.1 & 25.4 \\
\hline$\omega\left(\mathrm{s}^{-1}\right)$ & \multicolumn{2}{|c|}{3.1} & 2.81 & 2.77 & 1.58 & 1.60 & 1.59 \\
\hline$\delta$ & \multicolumn{2}{|c|}{3.1} & 3.3 & 3.5 & 1.13 & 1.13 & 1.12 \\
\hline$S t$ & 503 & 1125 & 10000 & 16400 & 505 & 1143 & 2057 \\
\hline$x_{0 \min }$ & 3.3 & 3.0 & 6.6 & 2.8 & \multicolumn{3}{c|}{$\sim 1$} \\
\hline$x_{0 \max }$ & 14 & 16 & 33 & 37 & 11 & 22 & 22 \\
\hline$R e_{\min }$ & 25 & 32 & 400 & 220 & 40 & 60 & 80 \\
\hline$R e_{\max }$ & 107 & 166 & 2000 & 3000 & 436 & 666 & 876 \\
\hline
\end{tabular}

are passive with the probe particle motion due to random thermal fluctuations i.e. to Brownian motion.

In 2005, Berg-Sørensen and Flyvbjerg [20] have shown that the study of the Brownian motion of a microsphere trapped in a viscous fluid by optical tweezers can be used to prove that the "thermal noise that drives the Brownian particle is not white, as in Einstein's simple theory [but] slightly coloured, due to hydrodynamics ". This effect is due to the fact that the hydrodynamic force given by eq. (4) is frequency-dependent because of the terms involving the viscous penetration depth $\delta=\sqrt{2 \eta / \rho \omega}$. Such an experiment was made in 2011 by Franosch et al. [21] in 2011 and by Jannasch et al. [22]. This type of experiments was reviewed by $\mathrm{Li}$ and Raizen [23]. These very nice experiments are in agreement with Stokes result giving the hydrodynamic force acting on a sphere and, because of the very small radius of the sphere used in these experiments, $a \approx 1 \mu \mathrm{m}$, and of the small velocities of the order of $10^{-3} \mathrm{~mm} / \mathrm{s}$, the Reynolds number is very small, $R e \sim 3 \times 10^{-3}$ (calculation done with the kinematic viscosity $\nu=\eta / \rho=5 \times 10^{-7} \mathrm{~m}^{2} / \mathrm{s}$ used by Franosch et al. [21]). However, precisely because of the very small radius of the spheres, the frequency for which $\delta \approx a$ is large, $\omega \approx 10^{6} \mathrm{~s}^{-1}$ : it seems very difficult to study with this type of experiment the case with $a$ considerably larger than $\delta$, especially because $\delta \propto 1 / \sqrt{\omega}$. 


\subsection{Comparison of these two types of tests}

It can be concluded that: with micron-sized particles the domain Re $\ll 1$ is easily observed but the particle size limits considerably the range of St number that can be explored.

with macroscopic objects it is far from easy to obtain small Re numbers but the main advantage is the large range of St numbers which is easily available.

\section{Our experiment}

\subsection{Principle of the experiment}

We measure the oscillation amplitude $x_{0}(t)$ of a pendulum as a function of the time $t$. The drag force being proportional to the velocity, $x_{0}(t)$ decreases exponentially. The drag force is deduced from the measured damping time constant $\tau_{\text {exp }}$ and the calculated energy of the pendulum. Non-hydrodynamic effects also induce energy losses: they are due to the anelastic behavior of the wire or to recoil of the support. Both effects appear because the force exerted by a flexible object is not in phase with its deformation. The theory of anelasticity $[24,25]$ gives a microscopic explanation based on heat transfer between the parts of a spring which are either heated or cooled by compression or expansion. Recoil losses [26] occur because the resonance frequencies of the pendulum support are not sufficiently large with respect to the pendulum frequency. The hydrodynamic and the non-hydrodynamic friction forces add their damping effects so that we can write

$\frac{1}{\tau_{\exp }}=\frac{1}{\tau_{h}}+\frac{1}{\tau_{n h}}$

where $\tau_{h}$ is the value of the damping time due to hydrodynamic forces only and $\tau_{n h}$ the value of this time due to non-hydrodynamic forces only. A measurement of the damping time constant with the pendulum at a pressure smaller than $10^{-5}$ mbar gives the value of $\tau_{n h}$, because the gas friction force at such a low pressure is negligibly small [26]. With the measured values of $\tau_{\text {exp }}$ and $\tau_{n h}$ and using eq. (11), we calculate $\tau_{h}$ from which we deduce the air friction force.

\subsection{The pendulum}

As the damping time constant $\tau_{\exp }$ increases with the pendulum mass, we have built light pendulums to limit the duration of the experiments. The pendulum body is a plastic spherical shell suspended by a piano string wire. The spherical shell is made of two hemispheres, $\sim 1 \mathrm{~mm}$ thick, that clip together and its suspension ring has been ground off [27]: the spheres thus formed are almost perfect with very small variations of their diameter (see appendix C). The suspension wire is glued at its top in a brass rod clamped in the pendulum support and at its bottom in a $3 \mathrm{~mm}$-diameter threaded brass rod. A $10 \mathrm{~mm}$-long nut and a threaded aluminium alloy cylinder are screwed on the threaded rod, thus clamping the upper hemisphere. A $0.06 \mathrm{~cm}^{3} \mathrm{NdFeB}$ magnet, fixed in this cylinder, is used to excite the pendulum oscillation thanks to the force exerted by a magnetic field gradient. Figure 1 shows some details of the pendulum and introduces the notations used to describe its motion [28]. For each pendulum, the dimensions and the oscillation frequency are given in table 3 ). The calculated values of the viscous penetration depth $\delta$ and of the Stokes number $S t$ are collected in table 4).

Finally, the pendulum is in a vacuum-tight cylindrical vessel (internal diameter $250 \mathrm{~mm}$ and height 800 $\mathrm{mm}$ ) which is clamped on a heavy optical table. The components of the shadow detector are fixed on this table. For the experiments at atmospheric pressure, this vessel protects the pendulum from air currents. This vessel is connected by a flexible pipe to an Edwards T85 pumping station made of a dry primary pump and a turbo pump and the residual pressure achieved after 24 hours of pumping is $\approx 4 \times 10^{-6}$ mbar.

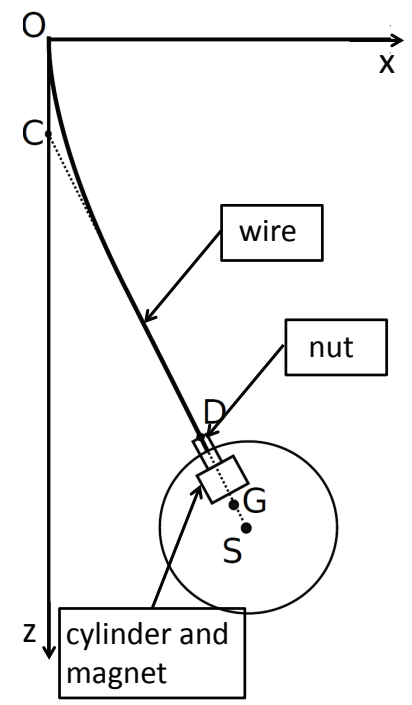

Fig. 1 Schematic drawing of the pendulum. The suspension wire is fixed in $O$ in the support and in $D$ in the pendulum body. $G$ is the center of mass of the body and $S$ is the center of the sphere. $C$ is the center of rotation of the pendulum body and $C$ would coincide with $O$ if the wire rigidity was negligible. The oscillation amplitude $x_{0}$ has been grossly exaggerated with respect to the experimental values $x_{0} \leq 0.6$ $\mathrm{mm}$ while the wire length $O D$ is $l_{W}=404 \pm 0.5 \mathrm{~mm}$. 


\subsection{The shadow detector}

We measure the displacement of the pendulum in the $\mathbf{x}$ direction with a shadow detector. This device measures the intensity of a light beam partly intercepted by the moving object, which is the nut located at the top of the sphere. The light beam is produced by a He-Ne laser with a beam expander and its power is $\sim 1 \mathrm{~mW}$. The transmitted intensity is measured by a photodiode with a current to voltage amplifier.

The detector sensitivity has been measured, using a knife edge on a micrometric translation: the signal as a function of the knife edge position is well represented by an error function, as expected for a Gaussian $\mathrm{TEM}_{00}$ beam. From this fit, we deduce the local radius $w \sim 2.8$ $\mathrm{mm}$ of the laser beam and the detector sensitivity $\sim 10^{3}$ $\mathrm{V} / \mathrm{m}$. From the $w$-value, we calculate that the deviation from linearity of this detector is equal to $5 \%$ for a displacement from the beam center equal to $0.16 w \approx 0.44$ $\mathrm{mm}$. As the maximum oscillation amplitude is close to $0.6 \mathrm{~mm}$, this non-linearity has very small effects on the measurement of the damping time constant $\tau$. We have verified that the time constant is modified by less than $1 \%$ if we suppress from the fit the first part of the amplitude record for a duration comparable to $\tau$ (this part is most sensitive to the detector non-linearity). Finally, the measured noise level is $\sim 3 \times 10^{-4} \mathrm{~V} / \sqrt{\mathrm{Hz}}$ at the pendulum frequency $\sim 0.8 \mathrm{~Hz}$. As a consequence, the displacement sensitivity is $\sim 3 \times 10^{-7} \mathrm{~m} / \sqrt{\mathrm{Hz}}$.

\subsection{Experimental protocol}

We excite the pendulum oscillation along the $\mathbf{x}$-axis by the force produced by a magnetic field gradient acting on the magnet. The magnetic field is produced by a coil located outside of the vessel enclosing the pendulum. This coil is powered by a voltage delivered by the computer. This voltage, which oscillates at the pendulum frequency, is applied during a time period chosen to produce an initial oscillation amplitude $x_{0}(0) \approx 0.6 \mathrm{~mm}$ for the spheres with a radius $a \leq 30 \mathrm{~mm}$. For the larger spheres, because of the larger drag force, the available force is not sufficient and the initial amplitude is reduced $x_{0}(0) \approx 0.3 \mathrm{~mm}$ if $a=50 \mathrm{~mm}$. The shadow detector signals are digitized at $40 \mathrm{~Hz}$ by a 16 -bit digitizer for a duration $\approx 7 \tau$ for the experiments at atmospheric pressure and only $\approx 4 \tau$ for the experiments under vacuum, because $\tau$ is considerably larger. At the end of the record, the process starts again.

\section{Data analysis}

\subsection{Extraction of the damping time constant $\tau$}

The recorded signal $V(t)$ is filtered at the pendulum frequency by a sliding Fast Fourier Transform over 2048 data points i.e. $\approx 51 \mathrm{~s}$. This filter reduces the noise but its averaging effect should not modify the damping time constant. If we call $V\left(t_{1}\right)$ the amplitude of the oscillating term, this filter gives an average value noted $\langle V(t)\rangle$ defined by

$$
\langle V(t)\rangle=\frac{1}{T} \int_{t-T / 2}^{t+T / 2} V\left(t_{1}\right) d t_{1}
$$

If $V\left(t_{1}\right)=V(0) \exp \left(-t_{1} / \tau\right)$, then $\langle V(t)\rangle \propto \exp (-t / \tau)$, which proves that the average of an exponential is an exponential with the same time constant. Using the detector sensitivity, we deduce from the filtered voltage the oscillation amplitude $x_{0}(t)$, which is expected to decay exponentially.

However, the vibrations of the laboratory, which are a stochastic function of time, modify the end of the decay [29]. We take this effect into account by fitting $x_{0}(t)$ by the function

$x_{0}(t)=x_{1} \exp (-t / \tau)+x_{2}$

where $x_{2}$ measures the mean value of the effect of the vibrations during the end of the record. For all our measurements, we find $\left|x_{2}\right| \leq 0.5 \mu \mathrm{m}$. In order to take advantage of the good signal-to-noise ratio of our measurements, we do not fit $x_{0}(t)$ but $\ln \left[x_{0}(t)\right]$ : this choice increases the weight of the data for $t \gg \tau$. As shown in fig. 2, this function represents very well the decay of $x_{0}(t)$ and the fit provides a measurement of the time constant $\tau$ with a small statistical uncertainty. As the initial value of the Reynolds number $R e$ is not smaller than 1 , we have tested the variation of the time constant $\tau$ if we fit only the part of the collected data with an amplitude $x_{0}(t)$ smaller than a fraction $\alpha$ of $x_{0}(0)$. If $0.2<\alpha<0.9$, the fitted value of $\tau$ is very stable, with variations below $1 \%$; if $\alpha<0.2, \tau$ increases and we attribute this behavior to the persistent oscillation due to the laboratory vibrations which modify the end of the amplitude damping. We have chosen the value of $\tau_{\text {exp }}$ equal to the average of the values over the range $0.2<\alpha<0.9$.

Because of the perturbation of the amplitude damping by the laboratory vibrations, we consider that the final amplitude is equal to $x_{\text {final }} \approx 3 \mu \mathrm{m}$, this last value being chosen because the damping is exponential as long as $x_{0}(t) \gg\left|x_{2}\right| \leq 0.5 \mu \mathrm{m}$. we will use the value of $x_{\text {final }}$ to evaluate the final value of the Reynolds number. 


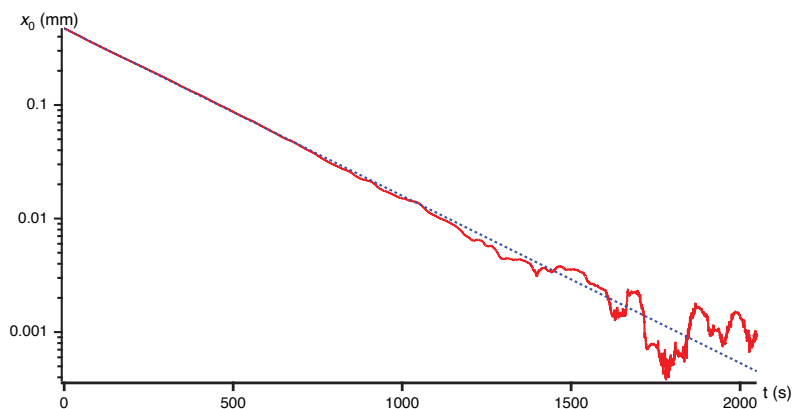

Fig. 2 The amplitude $x_{0}(t)(\mathrm{mm})$ is plotted as a function of the time $t(\mathrm{~s})$ : measured values (full red line) and their best fit (dotted blue line). The semi-logarithmic plot proves that the agreement of the fit extends until the $x_{2}$ term is no more negligible.

\subsection{Measured values of $\tau_{\exp }$ and $\tau_{n h}$}

For each pendulum, we have collected about 50 measurements of $\tau_{\exp }$ in a preliminary set-up operating only at atmospheric pressure, a similar number in the present set-up at atmospheric pressure and only about 5 measurements of $\tau_{n h}$ : this smaller number is due to the fact that the experiments under vacuum are considerably longer, up to $45000 \mathrm{~s}$, because of the larger value of $\tau_{n h}$. In table 2 , we collect the average values $\tau_{\exp 1}$ measured with the preliminary set-up and the average values $\tau_{\exp 2}$ measured with the present set-up. As these values are in agreement, we use their mean as the final value of $\tau_{\text {exp }}$ with an uncertainty equal to half their difference or to $2 \mathrm{~s}$, whichever is larger. We also give the average value of $\tau_{n h}$ measured under vacuum and the value of $\tau_{h}$.

Table 2 For each sphere, we give the rounded value in millimeters of its radius $a$, the measurements in seconds of the damping time constant measured under atmospheric pressure, $\tau_{\exp 1}$ in the preliminary set-up and $\tau_{\exp 2}$ in the set-up described above, their mean $\tau_{\exp }, \tau_{n h}$ measured under vacuum and $\tau_{h}$ calculated thanks to eq. (11)

\begin{tabular}{|c|c|c|c|c|}
\hline$a$ & $\tau_{\exp 1}$ & $\tau_{\exp }$ & $\tau_{n h}$ & $\tau_{h}$ \\
& $\tau_{\exp 2}$ & & & \\
\hline 15 & $435 \pm 1.6$ & $430 \pm 5$ & $7302 \pm 435$ & $456.9 \pm 6$ \\
& $425 \pm 2.0$ & & & \\
\hline 25 & $296 \pm 0.5$ & $292 \pm 4$ & $8157 \pm 296$ & $302.8 \pm 4$ \\
& $288 \pm 3.2$ & & & \\
\hline 30 & $262 \pm 0.4$ & $261 \pm 2$ & $9281 \pm 262$ & $268.6 \pm 2$ \\
& $260 \pm 2.6$ & & & \\
\hline 40 & $226 \pm 0.3$ & $228 \pm 2$ & $11997 \pm 226$ & $232.4 \pm 2$ \\
& $230 \pm 0.9$ & & & \\
\hline 50 & $233 \pm 0.2$ & $237 \pm 4$ & $13982 \pm 233$ & $241.1 \pm 4$ \\
& $241 \pm 2.0$ & & & \\
\hline
\end{tabular}

\section{Connection between the drag force and the damping time constant}

Here we present a simplified calculation with more details in Appendix C. In the absence of non-hydrodynamic forces, the pendulum quality factor $Q$ defined by $Q \equiv$ $\omega \tau_{h} / 2$ is also given by $Q=\Delta E_{h} /\left(2 \pi E_{t o t}\right)$ where $E_{t o t}$ is the pendulum total energy and $\Delta E_{h}$ is the energy lost during one oscillation period because of hydrodynamic forces. We first consider that the drag force $F_{x}^{\prime}$ is acting only on the sphere and we introduce a parameter $\beta_{S}$ defined by $F_{x}^{\prime}=-\beta_{S} v_{x}(t)$. The velocity of the sphere center is written $v_{x}(t)=v_{m}(t) \sin (\omega t)$, with $v_{m}(t) \approx-\omega x_{0}(t)$. The energy lost $\Delta E_{h}$ is given by

$\Delta E_{h}(t)=\pi \beta_{S} v_{m}^{2}(t) / \omega$.

If we add the contribution of the wire (see Appendix $\mathrm{B}$ ), we get the theoretical value $\beta_{t h}$ which is the sum of the effects of the drag forces on the sphere $\beta_{S}$ and on the suspension wire $\beta_{W}$, i.e. $\beta_{t h}=\beta_{S}+\beta_{W}$ with the following values

$$
\begin{aligned}
\beta_{S} & =\beta_{S 1}+\beta_{S 2} \\
\beta_{S 1} & =6 \pi \eta a \text { and } \beta_{S 2}=6 \pi \eta a^{2} / \delta \\
\beta_{W} & =2 \pi \eta\left(\frac{R}{\delta}\right)^{2} k^{\prime} \frac{l_{W}^{3}}{3 l_{S}^{2}} .
\end{aligned}
$$

$k^{\prime}$ is given by eq.(7), $Z$ being calculated with the wire radius $R ; l_{W}$ is the wire length and $l_{S}$ the distance from the top of the wire to the sphere center.

If we treat the pendulum as a rigid compound pendulum of moment of inertia $I$, the maximum angular velocity is equal to $v_{m}(t) / l_{S}$ where $l_{S}$ is the distance from the sphere center to the top of the suspension wire. The total energy $E_{t o t}$ is

$E_{t o t}(t)=I v_{m}^{2}(t) /\left(2 l_{S}^{2}\right)$

Using the two definitions of the quality factor $Q$, we deduce the relation between the coefficient $\beta$ and the damping time constant $\tau_{h}$. We note $\beta_{\text {exp }}$ the experimental value of $\beta$ given by

$\beta_{\text {exp }}=\frac{2 I}{l_{S}^{2} \tau_{h}}$

Because the suspension wire is not straight, the calculations of $\Delta E_{h}$ and $E_{t o t}$ are more complex (see Appendix C).

\section{Modification of Stokes' force due to fluid confinement}

If the fluid is confined, the drag force is increased because the velocity gradient is larger. Stokes [1] has calculated this effect in the case of the confinement by 
an external sphere of radius $b$ and, if $(b-a) \gg \delta$, the dependence of the drag force with $a, b$ and $\delta$ is given by his equation (61). With Stokes' notations, the drag force is written $F_{x}^{\prime}=-M^{\prime} k^{\prime} \omega d x / d t$, where $M^{\prime}$ is the mass of the displaced fluid $M^{\prime}=4 \pi a^{3} \rho / 3$, and $k^{\prime}(a, b, \delta)=\operatorname{Im}[K(a, b, \delta)]$ with $K(a, b, \delta)$ equal to

$$
\begin{aligned}
K(a, b, \delta)= & 1-\frac{3 b}{m^{2} a^{2}} \times \\
& \frac{\left(m^{2} a^{2}+3 m a+3\right)\left(m^{2} b^{2}-3 m b+3\right)}{b\left(m^{2} b^{2}-3 m b+3\right)-a\left(m^{2} a^{2}+3 m a+3\right)}
\end{aligned}
$$

where $m=(1+i) / \delta$. Using Mathematica, we have calculated the development of the ratio $k^{\prime}(a, b, \delta) / k^{\prime}(a, \infty, \delta)$ in powers of $(a / b)$ and we reproduce here only the first non-vanishing term

$$
\frac{k^{\prime}(a, b, \delta)}{k^{\prime}(a, \infty, \delta)}=1+\left(2+\frac{3 \delta}{a}\right)\left(\frac{a}{b}\right)^{3}+\mathcal{O}\left(\left(\frac{a}{b}\right)^{4}\right)
$$

In our experimental conditions, the viscous penetration depth $\delta$ is close to $2.5 \mathrm{~mm}$ and $\delta \ll a$. The first nonvanishing term of the confinement correction varies like $2(a / b)^{3}$. This correction is not negligible: if the cylindrical vessel enclosing the pendulum is equivalent to a sphere of radius $b=150 \mathrm{~mm}$, the numerical value of the ratio $k^{\prime}(a, b, \delta) / k^{\prime}(a, \infty, \delta)$ is 1.102 for $a=50 \mathrm{~mm}$ (for this calculation, we have used eq. (18) and not eq. (19) because the higher-order terms of the expansion are not negligible).

\section{Experimental results and discussion}

8.1 Comparison of the experimental and theoretical values of $\beta$

Figure 3 compares the theoretical and experimental values of $\beta$ (these values are listed in table 4 of Appendix C). The theoretical curve $\beta_{t h}$ takes into account confinement of the air by the vessel enclosing the pendulum, assuming that it is equivalent to a sphere of radius $b=140 \mathrm{~mm}$ : we choose this value because it is reasonable for a cylinder of radius $125 \mathrm{~mm}$ and also because it gives the best fit to the measured value $\beta_{\text {exp }}$. The experimental values $\beta_{\exp }$ exhibit very clearly the quadratic dependence upon the sphere radius $a$ predicted by Stokes. Moreover, the experimental and theoretical values are in very good agreement.

\subsection{Discussion of our theoretical evaluation of Stokes' force}

We discuss the effects which limit the accuracy of our evaluation of the coefficient $\beta_{t h}$ :

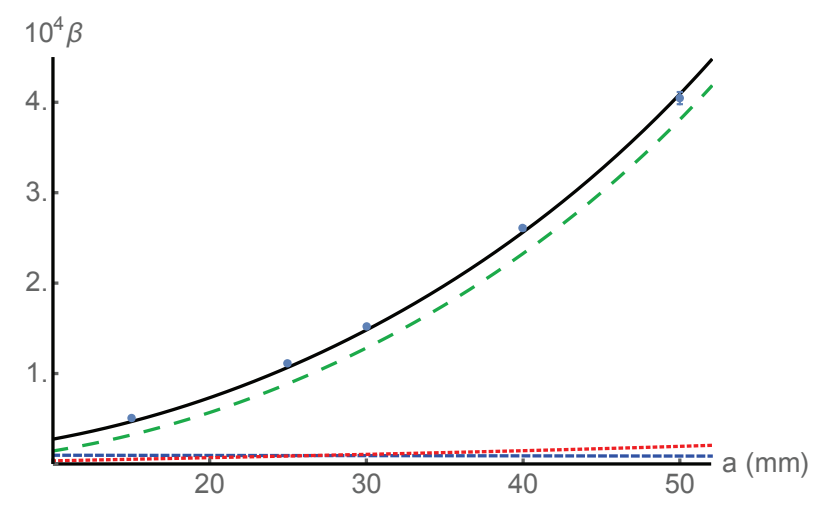

Fig. 3 The experimental and theoretical values of $\beta=$ $-F_{x}^{\prime} / v_{x}$ in $\mathrm{kg} / \mathrm{s}$ are plotted as a function of the sphere radius $a$. The blue dots represent the experimental values $\beta_{\text {exp }}$, with their error bars. The curves represent the theoretical value of $\beta_{S 1}$ (red dotted curve), $\beta_{S 2}$ (long dashed green curve), $\beta_{W}$ (short dashed blue curve) and their sum $\beta_{t h}$ (full line black curve). The quantities $\beta_{S 1}$ and $\beta_{S 2}$ include the correction by confinement calculated for a sphere of radius $b=140$ $\mathrm{mm}$, chosen as it gives the best fit to the measurements. The theoretical functions take into account the variation of the viscous penetration depth $\delta$ with the sphere radius $a$ because the pendulum frequency is a function of $a$, namely $\omega /(2 \pi) \mathrm{mHz}=878-2.42 a$ with $a$ in $\mathrm{mm}$.

- Validity of Stokes' calculation. This validity requires the oscillation amplitude $x_{0}(t)$ to be small with respect to $a$ and $\delta$ for the sphere and $R$ and $\delta$ for the wire. For the sphere, the ratio $x_{0}(0) / a$ covers the range 0.03-0.01 when $a$ increases and the condition $x_{0}(t) \ll a$ is very well fulfilled. As $\delta \approx 2.5 \mathrm{~mm}$, the ratio $x_{0}(t) / \delta$ decreases from 0.2 down to $10^{-3}$ during the damping and the condition $x_{0}(t) \ll \delta$ is also very well fulfilled during the largest part of the damping. For the wire, the amplitude varies along the length of the wire and is maximum at its bottom where it is equal to the one of the sphere. The condition $x_{0}(t) \ll R$ is not fulfilled during the initial part of the damping as $x_{0}(0) \approx 0.6$ $\mathrm{mm}$ while $R \approx 0.25 \mathrm{~mm}$. Following Berg et al. [7], the force increases but the correction is small. It would be difficult to make a quantitative evaluation of this effect because the force is proportional to the velocity with a proportionality coefficient which is a function of the amplitude and as a consequence of the time and of the point on the wire.

- Drag force on the wire. In eq. (15), the effects of the drag force on the sphere and on the wire are simply added. As the lowest part of the wire is in the fluid dragged by the sphere, this calculation overestimates the length of the wire and the energy loss but the relative error should be small. As the velocity field has two length scales $\delta$ and $a$, the part of this correction comparable to $\delta$ is fully negligible but the part comparable to $a$ is more important. However, this last correction 
is small for the smallest spheres while, for the largest spheres, $\beta_{W}$ is a small fraction of $\beta_{t h}$ : in all cases, this correction is a very small fraction of $\beta_{t h}$.

- Ambient air. The calculation of $\beta_{t h}$ used $\eta=$ $18.303 \times 10^{-6}$ Pa.s [30] and $\rho=1.175 \mathrm{~kg} / \mathrm{m}^{3}$ corresponding to the mean Toulouse atmospheric pressure $\approx 0.995 \times 10^{5} \mathrm{~Pa}$ and to the temperature $T=295 \mathrm{~K}$. The pressure and temperature, not controlled during the experiments, vary typically by $\pm 2 \times 10^{3} \mathrm{~Pa}$ and by $\pm 2 \mathrm{~K} . \eta$ is independent of the pressure and varies like $T^{0.787}$ while the density $\rho$ varies proportionally to the ratio pressure/temperature. The associated errors are $\pm 0.53 \%$ for $\eta, \pm 2.7 \%$ for $\rho, \pm 1.6 \%$ for $\delta$ and $1.1 \%$ for the ratio $\eta / \delta \propto \sqrt{\eta \rho}$ to which the dominant term $\beta_{S 2}$ is proportional. Air humidity [31] has little effect on $\eta$ which decreases by $0.4 \%$ when going from dry air to saturation at $298 \mathrm{~K}$. We estimate that the resulting error bar on $\beta_{t h}$ is $\pm 2 \%$.

None of the effects discussed here can modify noticeably the theoretical value $\beta_{t h}$. The largest effect which is not rigorously described is the correction due to the fluid confinement.

\section{Conclusion}

We have tested Stokes' result for the drag force on an oscillating sphere by experiments in the validity domain of Stokes' calculation with the Reynolds number $R e \ll 1$. All previous similar tests with macroscopic objects have been made with large values of the Reynolds number, $R e \geq 20$. Some recent experiments [21,22] using microrheology techniques have tested Stokes' result with very small values of the Reynolds number $R e \sim 10^{-3}$ but these experiments are presently limited to the domain where the viscous penetration depth $\delta$ is larger or comparable to the sphere radius $a$ i.e. to small values of the Stokes number. In our experiments, the Reynolds number $R e$ decreases from an initial value $5-10$ down to a final value $0.03-0.09$, depending of the sphere radius and the Stokes number covers the range 150-1500 (see table 4 ). The oscillation amplitude $x_{0}$ is always very small with respect to the sphere radius $a$ and it is also small with respect to the viscous penetration depth $\delta$. We have thus obtained the following results:

- when the sphere radius $a$ is larger than the viscous penetration depth $\delta$, the measured drag force has a quadratic dependence with the sphere radius $a$, as predicted by Stokes, and its value is in very good agreement with Stokes' result: the differences between our measurements and our theoretical evaluation of the drag force coefficient $\beta$ are of the order of a few percents;

- because of the confinement of the fluid by a cylindrical vessel, it is necessary to include a correction. This effect, which increases the drag force, was calculated by Stokes in 1851 in the case of the confinement by a sphere of radius $b$. With our experimental parameters, the leading term of this correction is in $2(a / b)^{3}$. We have taken into account this effect, assuming that the vessel enclosing the pendulum is equivalent to a sphere of radius $b=140 \mathrm{~mm}$. Thanks to this correction, our measurements are in very good agreement with the theoretical values.

Acknowledgements We want to thank D. Castex, E. Panader, S. Faure, L. Polizzi and W. Volondat for their help with the experiment. We have benefited from very interesting discussions with P. Ern, M. Nicolas, F. Charru and with R.F. Berg, who also made a critical reading of a first version of our MS. Financial supports from CNRS INP, CNRS MI DEFI and Université Paul Sabatier are gratefully acknowledged.

\section{Conflict of interest}

The authors declare that they have no conflict of interest.

\section{A Quasi-degeneracy of the normal modes of our pendulum}

The pendulum has an almost perfect cylindrical symmetry around the wire axis. Such a pendulum has two normal modes with two oscillation frequencies which would be exactly equal if the cylindrical symmetry was perfect and which are slightly different because this symmetry is weakly broken. In our experiment, we observe a weak symmetry breaking: a possible explanation would be an elliptic cross section of the suspension wire but we have found that the dominant contribution is due to the interaction of the magnet carried by the pendulum with the gradient of the laboratory magnetic field. We treat of this problem, in the case of a simple pendulum of mass $m$. Its kinetic energy term $E_{K}$ is isotropic and the anisotropy comes from the potential energy term $E_{P}$

$$
\begin{aligned}
& E_{K}=\frac{m}{2}\left[\left(\frac{d u}{d t}\right)^{2}+\left(\frac{d v}{d t}\right)^{2}\right], \\
& E_{P}=\frac{1}{2}\left[k_{u} u^{2}+k_{v} v^{2}\right] .
\end{aligned}
$$

Here, $\mathbf{u}$ and $\mathbf{v}$ are the axes which give a diagonal form to $E_{P}$ and $u$ and $v$ the coordinates of the pendulum center of mass in this coordinate system. The normal modes correspond to oscillations along the $\mathbf{u}$ and $\mathbf{v}$ axes with the angular frequencies

$\omega_{u}=\sqrt{k_{u} / m}$ and $\omega_{v}=\sqrt{k_{v} / m}$.

We consider the displacement of the pendulum in the $\mathbf{x}$ direction. In the general case, the pendulum is in a superposition of the two normal modes

$u=u_{0} \cos \left(\omega_{u} t+\varphi_{u}\right)$ and $v=v_{0} \cos \left(\omega_{v} t+\varphi_{v}\right)$

We note $\theta$ the angle between the $\mathbf{u}$ and $\mathbf{x}$ axes and $(\pi / 2)-\theta$ the angle $\mathbf{v}$ and $\mathbf{x}$. The position of the pendulum along the $\mathbf{x}$-axis

$x=x_{u} \cos \left(\omega_{u} t+\varphi_{u}\right)+x_{v} \cos \left(\omega_{v} t+\varphi_{v}\right)$ 
with $x_{u}=u_{0} \cos \theta$ and $x_{v}=v_{0} \sin \theta$. We introduce the phases $\psi_{+}$and $\psi_{-}$defined by

$\psi_{+}=\left[\left(\omega_{u}+\omega_{v}\right) t+\varphi_{u}+\varphi_{v}\right] / 2$

$\psi_{-}=\left[\left(\omega_{u}-\omega_{v}\right) t+\varphi_{u}-\varphi_{v}\right] / 2$

$\psi_{+}$describes the fast oscillation while $\psi_{-}$describes the envelope of the beats.

$x=\left(x_{u}+x_{v}\right) \cos \psi_{-} \cos \psi_{+}+\left(-x_{u}+x_{v}\right) \sin \psi_{-} \sin \psi_{+}$

The amplitude of the fast oscillation is a function of the time $t$. It is given by the modulus of the Fourier component of frequency $\omega_{+}=\left(\omega_{u}+\omega_{v}\right) / 2$ :

$\tilde{x}_{\omega_{+}}(t)=\sqrt{x_{u}^{2}+x_{v}^{2}+2 x_{u} x_{v} \cos \left(\omega_{-} t+\varphi_{-}\right)} \exp (-t / \tau)$,

where $\omega_{-}$and $\varphi_{-}$are the frequency and the initial phase of the beat pattern. We have taken into account damping by adding the factor $\exp (-t / \tau)$. This is correct if the damping forces are equal for the two normal modes: this assumption is excellent as the pendulum has an almost perfect cylindrical symmetry.

We now apply these results to the treatment of our experimental data. The measured amplitude $x_{0}(t)$ is an average of $\tilde{x}_{\omega_{+}}(t)$, because of the use of the sliding Fast Fourier Transform. When the pendulum is under vacuum, the damping time constant $\tau$ is very long and we observe beats which are fitted by the following equation

$x_{0}(t)=\sqrt{A+B \cos \left(\frac{2 \pi t}{T_{0}}+\phi\right)} \exp (-t / \tau)$.

This equation is equivalent to eq. (27) but better suited for a fit. In this case, even if we record the oscillation for a long time, the final amplitude is not very small and there is no need to include a term representing the mean effect of the vibrations of the experiment. Fig. 4 presents an example of the recorded data and its fit by eq. (28).

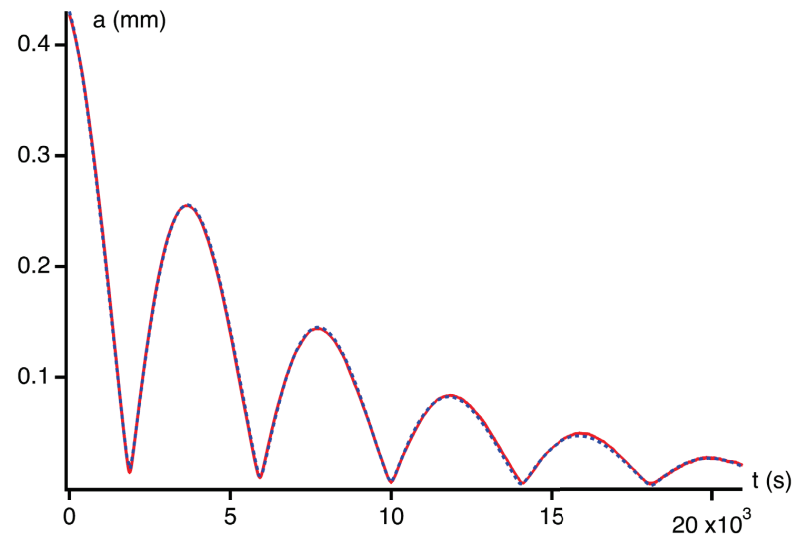

Fig. 4 Damping of the oscillation of the sphere of radius $a=15 \mathrm{~mm}$ under vacuum. The measured amplitude $x_{0}(t)$ is plotted as a function of the time $t$ (s) (full red line) and its fit using eq. (28) (dashed blue line). The damping time constant $\tau$ and the beat period $T_{0}$ given by the fit are $\tau=7174 \mathrm{~s}$ and $T_{0}=4061$. The amplitudes $A$ and $B \mathrm{~mm}$ are almost equal in this experiment: this condition, which maximizes the beat contrast, occurs if $\theta \approx \pm \pi / 4$.
In a preliminary set-up, we measured the oscillation amplitude in two perpendicular directions with two shadow detectors measuring the amplitude $\tilde{x}_{\omega_{+}}(t)$ and $\tilde{y}_{\omega_{+}}(t)$. By taking the square root of the sum of the squares of these two amplitudes, we eliminated the effect of the beats and the damping was exponential. The results of this preliminary experiment appear in table 2 in the column $\tau_{\exp 1}$.

In the present set-up, we measure only the amplitude $\tilde{x}_{\omega_{+}}(t)$. When the pendulum is at atmospheric pressure, the damping time constant $\tau$ is considerably shorter than under vacuum. We do not observe beats and we use eq. (13) to fit the measured amplitude. This technique was successful for all the spheres except for the smallest one with a radius $a=15 \mathrm{~mm}$. For this sphere, the measured damping time constant varied noticeably when we reduced the fraction $\alpha$ of the initial amplitude. We think that this was due to undetected beats: the beat period $T_{0} \approx 4000 \mathrm{~s}$ (see fig. 4 ) is sufficiently large with respect to the damping time constant $\tau \approx 430$ s so that we do not observe beats for this sphere but the hidden beat pattern makes that the damping is not exponential (this effect appears to be negligible for larger spheres because the beat period $T_{0}$ increases when the pendulum mass increases and, at the same time, the damping time constant $\tau$ decreases as shown in table 2). For the sphere with $a=15 \mathrm{~mm}$, we have applied a dipolar magnetic field produced by a magnet in a geometry such that the excited oscillation is a normal mode and then the beat amplitude is negligible. This technique restored an exponential decay of the amplitude and we thus measured the value of $\tau_{\exp 2}$ for this sphere.

\section{B Calculation of the energy lost $\Delta E_{h}$}

We calculate the energy lost $\Delta E_{h}$ because of the hydrodynamic forces acting on the sphere and on the wire. The drag force on the sphere given by eq. (4) can be rewritten $F_{x}^{\prime}(t)=$ $-\beta_{S} v_{x}(t)$. The velocity of the sphere center being $v_{x}(t)=$ $v_{m}(t) \cos (\omega t)$, where $v_{m}(t)$ varies slowly with $t$, the work $\Delta E_{S}(t)$ done by this drag force during one period of oscillation $T=2 \pi / \omega$ is given by a straightforward integration

$\Delta E_{S}(t) \approx \beta_{S} \frac{\pi v_{m}^{2}(t)}{\omega}$

The drag force per unit length of the wire given by eq. (6) is used to calculate the force on each element $d z$ of the wire as if it was part of an infinite cylinder moving with a uniform oscillating velocity

$\frac{d F_{x}^{\prime}}{d z}=-\beta_{W} v_{x}(z, t)$ with $\beta_{W}=2 \pi \eta\left(\frac{R}{\delta}\right)^{2} k^{\prime}$

If the wire is approximated by a straight line, the velocity $v(z, t)$ is proportional to the distance $z$ and related to the velocity of the sphere center by

$v_{x}(z, t)=\frac{z}{l_{S}} v_{m}(t) \cos (\omega t)$

The work $\Delta E_{W}(t)$ done by the drag force on the wire during one period of oscillation is then given by integration over the length of the wire and over one period of oscillation

$\Delta E_{W}(t) \approx \beta_{W} \frac{l_{W}^{3}}{3 l_{S}^{2}} \frac{\pi v_{m}^{2}(t)}{\omega}$

We thus get

$\Delta E_{h}(t)=\Delta E_{S}(t)+\Delta E_{W}(t)=\beta \frac{\pi v_{m}^{2}(t)}{\omega}$ 


$$
\begin{aligned}
\text { with } \beta & =\beta_{S 1}+\beta_{S 2}+\beta_{W} \\
\beta_{S 1} & =6 \pi \eta a \text { and } \beta_{S 2}=6 \pi \eta a^{2} / \delta \\
\beta_{W} & =2 \pi \eta\left(\frac{R}{\delta}\right)^{2} k^{\prime} \frac{l_{W}^{3}}{3 l_{S}^{2}}
\end{aligned}
$$

In order to compare this theoretical value $\beta_{t h}$. to its experimental values $\beta_{\text {exp }}$, we will take into account the correction due to the fluid confinement given by eq. (18) and this correction is applied only to the sphere terms $\beta_{S 1}$ and $\beta_{S 2}$.

\section{Refined theory of the pendulum}

We have developed the theory of a flexible-beam pendulum [28] i.e. a rigid pendulum body suspended by a suspension spring, as used in traditional clocks. This theory predicts the existence of two resonances corresponding to different motions of the pendulum: we are interested here in the lowest frequency resonance which is associated to the usual pendular motion. The theoretical values of these two frequencies have been compared to experimental results [32] and the agreement is good, especially for the lowest frequency resonance, which is the one we are interested here. We refer the reader to our paper [28] for the equations which are used here to calculate the frequency and total energy of our pendulum. To apply this theory, we must know the masses of the pendulum components and their dimensions as well as the elastic properties of the suspension wire.

\section{C.1 Information on the pendulum and calculation of} its frequency

Figure 1 presents a schematic drawing of the pendulum. The free length $O D$ of the suspension wire is noted $l_{W}$. The distance $O S$, when the pendulum is at rest, is noted $l_{S}=l_{W}+$ $l_{N}+a$ where $l_{N}=10 \mathrm{~mm}$ is the nut length (see fig. 1 of our letter). The length $D G$ is noted $h$ and the gyration ratio of the pendulum body is noted $\rho$. We have weighted the various parts of the pendulum and measured the various dimensions to calculate the total mass $M$ of the pendulum, the distance $D G=h$ and the gyration ratio $\rho$ of the pendulum body. All these values are given in table 3 .

The pendulum suspension wire is a piano string of diameter $2 R=0.49 \pm 0.01 \mathrm{~mm}$, of length $l_{W}=404 \pm 0.5 \mathrm{~mm}$ and mass $m_{W}=0.60 \mathrm{~g}$. Moreover, the theory [28] which takes into account the bending rigidity of this wire requires the product $E I_{s}$ for the suspension wire, where $E$ is the Young modulus of the wire material and $I_{s}$ is the second moment of the area of its cross section, $I_{s}=\pi R^{4} / 4$. We have measured this quantity as in our previous work [28] by measuring the deflection of a portion of this wire initially horizontal by a series of small test masses. We thus get $E I_{s}=(6.17 \pm 0.07) \times 10^{-4} \mathrm{~N} . \mathrm{m}^{2}$, in good agreement with the calculated value $E I_{s}=(5.94 \pm 0.48) \times 10^{-4} \mathrm{~N} . \mathrm{m}^{2}$, using the value $E \approx 210 \mathrm{GPa}$ of Young's modulus for high carbon content steel (value from he website http://www.matweb.com/)

In our paper [28], the mass of the flexible beam was neglected with respect of the pendulum body and this was a good approximation in our experimental test [32]. It is no more the case in the present experiment, because the wire mass $(0.60 \mathrm{~g})$ is not negligible with respect to the other masses, especially for the smallest spheres. As a consequence, in the calculation of $\rho$ and $M$, we have included the contribution of the wire as if it was straight. From these parameters, we deduce the parameter $\kappa=\sqrt{M g /\left(E I_{S}\right)}$ and the dimensionless parameter $\chi=\kappa l_{W}$. Using the equations (23) and (24) of our paper [28], we calculate the frequency $\omega /(2 \pi)$ of its lowest resonance and the length $\lambda$. The agreement of the calculated frequency with its measured value is very good.

Table 3 For each sphere, we give the mean value of its radius $a$, with an uncertainty interval $2 \Delta a$ covering the variations of the diameter measured along 7 well-spaced directions, the total pendulum mass $M$ [33], the lengths $l_{S}$ and $h$ and the gyration ratio $\rho$. From these quantities and the quantity $E I_{s}$ for the suspension wire, we deduce the parameters $\kappa$ and $\chi=$ $\kappa l_{W}$. We compare the measured pendulum frequency to its calculated value. Finally, we give the calculated value of the distance $\lambda=C D$, which is substantially smaller than the wire length $l_{W}=404 \pm 0.5 \mathrm{~mm}$.

\begin{tabular}{|c|c|c|c|c|c|}
\hline$a(\mathrm{~mm})$ & 15.01 & 25.08 & 29.96 & 40.02 & 50.01 \\
\hline$\Delta a(\mathrm{~mm})$ & \pm 0.09 & \pm 0.03 & \pm 0.14 & \pm 0.06 & \pm 0.04 \\
\hline$M(\mathrm{~g})$ & 12.35 & 17.92 & 21.54 & 31.43 & 49.52 \\
\hline$l_{S}(\mathrm{~mm})$ & 429. & 439. & 444. & 454. & 464. \\
\hline$h(\mathrm{~mm})$ & 8. & 18. & 24. & 36. & 49. \\
\hline$\rho(\mathrm{mm})$ & 55. & 49. & 48. & 48. & 50. \\
\hline$\kappa\left(\mathrm{m}^{-1}\right)$ & 13.7 & 16.4 & 18.0 & 21.8 & 27.3 \\
\hline$\chi$ & 5.5 & 6.6 & 7.3 & 8.8 & 11.0 \\
\hline $\begin{array}{c}\text { Measured } \\
\text { frequency (Hz) }\end{array}$ & 0.845 & 0.817 & 0.806 & 0.782 & 0.758 \\
\hline $\begin{array}{c}\text { Calculated } \\
\text { frequency (Hz) }\end{array}$ & 0.843 & 0.817 & 0.805 & 0.780 & 0.757 \\
\hline$\lambda(\mathrm{mm})$ & 306. & 320. & 326. & 336. & 345. \\
\hline
\end{tabular}

\section{C.2 Calculation of the drag force coefficient $\beta$}

Using our theory of this type of pendulum [28], we calculate the total energy $E_{t o t}$ and the energy lost $\Delta E_{h}$ by the drag forces during one period of oscillation as a function of thedrag force coefficient $\beta$. In our calculation, we take into account the exact shape of the wire. The results of this calculation are given in table 4 . In this table, we also give the theoretical value $\beta_{t h}$. of this coefficient, calculated using eq. (33) and taking into account the correction for the fluid confinement given by eq. (18), assuming that the vessel enclosing the pendulum is equivalent to a sphere of radius $b=140 \mathrm{~mm}$.

Table 4 For each pendulum, this table gives the rounded value of the sphere radius $a$, the value of the viscous penetration depth $\delta$, the value of the Stokes number $S t$, the experimental value $\beta_{\text {exp }}$ deduced from the value of the damping time constant $\tau_{h}$ given in table 2 and the theoretical value $\beta_{t h}$ (see text).

\begin{tabular}{|c|c|c|c|c|}
\hline $\mathrm{a}(\mathrm{mm})$ & $\delta(\mathrm{mm})$ & $S t$ & $10^{6} \beta_{\exp } \mathrm{kg} / \mathrm{s}$ & $10^{6} \beta_{t h .} \mathrm{kg} / \mathrm{s}$ \\
\hline 15 & 2.42 & 153 & $50.9 \pm 0.7$ & 46.8 \\
\hline 25 & 2.46 & 415 & $111.4 \pm 1.4$ & 106.8 \\
\hline 30 & 2.48 & 584 & $151.9 \pm 1.1$ & 148.1 \\
\hline 40 & 2.52 & 1010 & $260.2 \pm 2.2$ & 256.4 \\
\hline 50 & 2.56 & 1530 & $403.7 \pm 6.7$ & 409.0 \\
\hline
\end{tabular}




\section{References}

1. G.G Stokes, "On the effect of the internal friction of fluids on the motion of pendulums", Transactions of the Cambridge Philosophical Society, vol IX, part II, 8-106 (1851).

2. L. Landau and E. Lifschitz, "Fluid mechanics", Pergamon, London (1959).

3. J.T. Stuart, Chap. VII, pp 347-408, in Laminar Boundary Layers, edited by L. Rosenhead, Oxford University Press (1963).

4. R.G. Hussey and P. Vujacic, "Damping correction for oscillating cylinder and sphere", Phys. Fluids 10, 96-97 (1967).

5. All the dimensionless quantities can be expressed as a function of $R e$ and $S t$. The ratio $x_{0} / a$ is equal to $K C / \pi$, where $K C$ is the Keulegan-Carpenter number [6].

6. G.H. Keulegan and L.H. Carpenter, "Forces on cylinders and plates in an oscillating fluid," Journal of Research of the National Bureau of Standards 60, 423-440 (1958).

7. R.F. Berg, M. Yao, and C.H. Panzarella, "Hydrodynamic force on a cylinder oscillating at low frequency," NASA/CR 2007 - 215050.

8. F. Baily, "On the correction of a pendulum for the reduction to a vacuum: together with remarks on some anomalies observed in pendulum experiments," Phil. Trans. R. Soc. Lond. 122 399-492 (1832).

9. J.T. Stuart and L. Woodgate, "Experimental determination of the aerodynamic damping on a vibrating circular cylinder", Phil. Mag. 46, 40-46 (1955).

10. V.K. Gupta, Gauri Shanker, and N.K. Sharma, "Experiment on fluid drag and viscosity with an oscillating sphere," Am. J. Phys. 54, 619-622 (1986).

11. D. Bolster, R.E. Hershberger, and R.J. Donnelly, "Oscillating pendulum decay by emission of vortex rings", Phys. Rev. E 81, 046317 (2010).

12. L.R. Laird, "On the period of a wire vibrating in a liquid", Phys. Rev Series I 7, 102-104 (1898).

13. H. Martin, "Uber tonhöhe und dämpfung der schwingungen von saiten in verschiedenen flüssigkeiten", Ann. Phys. Leipzig 4, 627-657 (1925).

14. C.A. Coulomb, "Expériences destinées à déterminer la cohérence des fluides et les lois de leur résistance dans les mouvements très lents," Mémoires de l'Institut National des Sciences et des Arts 3, 246-305 (1800).

15. R.E. Williams, "Oscillating cylinders and the Stokes paradox," (1972) PhD Louisiana State University, LSU Historical Dissertations and Theses. 2319

16. R.E. Williams and R.G. Hussey, "Oscillating cylinders and the Stokes' paradox", Phys. Fluids 15, 2083-2088 (1972) and erratum 19, 1652 (1976).

17. H. Tomlinson, "The coefficient of viscosity of air," Phil. Trans. 177, 767-799 (1886).

18. J. Jäger, B. Schuderer, and W. Schoepe, "Translational oscillations of a microsphere in superfluid helium", Physica B 210, 201-208 (1995).

19. M.L. Gardel, M.T. Valentine, and D.A. Weitz, "Microrheolog," in Microscale Diagnostic Techniques, Ed. K. Breuer (2005).

20. K. Berg-Sørensen and H. Flyvbjerg, " The colour of thermal noise in classical Brownian motion: a feasibility study of direct experimental observation," New J. Phys. 738 (2005).

21. Th. Franosch, M. Grimm, M. Belushkin, F. Mor, G. Foffi, L.o Forro, and S. Jeney, "Resonances arising from hydrodynamic memory in Brownian motion - The colour of thermal noise, " Nature, 478, 85 (2011).

22. A. Jannasch, M. Mahamdeh, and E. Schäffer, "Inertial effects of a small Brownian particle cause a colored power spectral density of thermal noise," Phys. Rev. Lett., 107, 228301 (2011)
23. T. Li and M.G. Raizen, "Brownian motion at short time scales," Ann. Phys. (Berlin) 525, 281-295 (2013).

24. C. Zener, "Internal friction in solids. I. Theory of internal friction in reeds," Phys. Rev. 52, 230-235 (1937).

25. C. Zener, "Internal friction in solids. II. General theory of thermoelastic internal friction," Phys. Rev. 53, 90-99 (1938).

26. G. Cagnoli, L. Gammaitoni, J. Hough, J. Kovalik, S. McIntosh, M. Punturo, and S. Rowan, "Very high $Q$ measurements on a fused silica monolithic pendulum for use in enhanced gravity wave detectors," Phys. Rev. Lett. 85, 2442-2445 (2000).

27. These spheres were ordered from http://www.ballkit.fr.

28. G. Dolfo and J. Vigué, "A more accurate theory of a flexible-beam pendulum", Am. J. Phys. 83, 525-530 (2015).

29. D. Paget, J. Winterflood, Li Ju, and D. Blair, "Improved technique for measuring high pendulum Q-factors", Meas. Sci. Technol. 13, 218-221 (2002).

30. E.W. Lemmon, R.T. Jacobsen, "Viscosity and thermal conductivity equations for nitrogen, oxygen, argon, and air", Int. J. Thermophysics 25, 21-69 (2004) and NIST software RefProp 9.0 (2010).

31. J. Kestin and J.H. Whitelaw, "The viscosity of dry and humid air", Int. J. Heat Mass Transfer. 7, 1245-1255 (1964).

32. G. Dolfo, D. Castex, and J. Vigué, "Damping mechanisms of a pendulum", Eur. J. Phys. 37, 065004 (2016).

33. The present work is a part of the PhD thesis of one of us (GD). We found that the electronic mass balance used to measure the masses of the pendulum components gave wrong values, $\approx 80 \%$ of the correct value. This error has been corrected and we have redone all the experiments. 